\title{
On a semiclassical model for ion-acoustic solitons in ultrarelativistic pair plasmas and its classical counterpart
}

\author{
Cite as: Phys. Plasmas 26, 062301 (2019); doi: 10.1063/1.5100244 \\ Submitted: 16 April 2019 • Accepted: 28 May 2019 • \\ Published Online: 17 June 2019

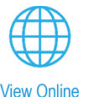 \\ Frank Verheest $^{1,2, a)}$ (iD and loannis Kourakis ${ }^{3, b)}$ (iD \\ AFFILIATIONS \\ ${ }^{7}$ Sterrenkundig Observatorium, Universiteit Gent, Krijgslaan 281, B-9000 Gent, Belgium \\ ${ }^{2}$ School of Chemistry and Physics, University of KwaZulu-Natal, Durban 4000, South Africa \\ ${ }^{3}$ Department of Sciences and Engineering, Sorbonne University Abu Dhabi, Abu Dhabi, United Arab Emirates \\ a)Electronic mail: frank.verheest@ugent.be

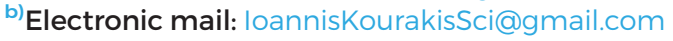

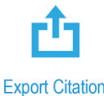

\begin{abstract}
Large ion-acoustic solitary waves are investigated in a multispecies plasma model consisting of warm positive ions in the presence of ultrarelativistic electrons and positrons, in a Sagdeev pseudopotential formalism. A parametric investigation determines existence regions in terms of fractional densities, temperature ratios, and soliton speeds. Various examples of pseudopotential functional forms, as well as those of the resulting soliton and electric field profiles, can then be generated numerically, and some typical illustrations have been included. Rather than adiabatic pressuredensity relations for the hot species, the classical nonrelativistic counterpart involves Boltzmann distributions, which differ qualitatively from the literature. Surprisingly, the soliton and electric field profiles show scant differences at the same compositional parameters between the two extremes even though the physical description of the hot species is radically different. A brief comparison has also been included between the fully nonlinear Sagdeev pseudopotential descriptions and their respective associated weak-amplitude limits (treated via a reductive perturbation technique) in which nonlinearities have been truncated to low powers of the electrostatic potential. Again, the soliton profiles are not radically different at comparable amplitudes, leaving the underlying physical reasons for such a similarity an open problem.
\end{abstract}

Published under license by AIP Publishing. https://doi.org/10.1063/1.5100244

\section{INTRODUCTION}

There is an ongoing interest in the propagation of nonlinear solitary waves in unusual plasma configurations, many of those in heliospheric and astrophysical settings. One area concerns acoustic-type solitons in electron-positron-ion (e-p-i) plasmas as in white dwarfs and magnetars, having ultrarelativistic thermodynamics. ${ }^{1-18}$ Pure pair plasmas, with their inherent symmetry in mass and charge between electron and positrons or equivalent descriptions in terms of positive and negative ions, can only support acoustic solitons if there is an imbalance in either thermodynamics or in undisturbed densities. ${ }^{19}$ In the latter case, a third charged species is needed to ensure an electrically neutral equilibrium.

Our interest in the study at hand focuses on a plasma fluid model often encountered in the literature, ${ }^{1,4,7}$ wherein an electron-positronion (e-p-i) configuration is adopted, with less positrons than electrons, in addition to positive ions, to ensure overall charge neutrality. For the sake of rigor and completeness let us add that, conversely, one might think of a model with less electrons than positrons, and thus with negative ions, in order to ensure charge balance in equilibrium. However, as will be indicated below, the latter configuration is mathematically equivalent to having positive ions, in view of the symmetry in charge and mass between the electrons and positrons, up to some necessary but trivial changes in polarities and normalization.

In several investigations of e-p-i plasmas with ultrarelativistic pressure-density relations, the classical nonrelativistic counterpart is also included, with adiabatic pressure-density relations. In the modeling of hot species, their inertial effects are often neglected in favor of a balance between pressure and electric forces, on the grounds of their thermal velocities being large compared to wave and/or soliton speeds. However, as a careful discussion shows, ${ }^{20}$ in the classical nonrelativistic case, one should use Boltzmann distributions, the adiabatic pressuredensity relations often used ${ }^{1,4,7,9,11-13}$ for hot inertialess species being then a poor description. This means that the transition from ultrarelativistic to classical nonrelativistic might be discussed as two opposite 
limits of the same formulation, $\gamma=4 / 3$ for ultrarelativistic as opposed to $\gamma=1$ for classical nonrelativistic hot species, but we prefer to deal with these opposites in separate sections below.

Treatments in terms of a general $\gamma$ in the pressure-density relations ${ }^{4}$ were considered of limited interest because many of the intermediate $\gamma$ values traditionally lacked an immediate physical interpretation. In a recent paper, ${ }^{21}$ however, it is proved that there is a two-way equivalence between polytropic pressure-density models and $\kappa$ distributions for the hot species. As $\kappa$ can assume a whole range of values, from Boltzmann to very nonthermal equations of state, so then can $\gamma$, although the $\kappa$ descriptions have usually remained in the classical sphere.

The purpose of this paper is to investigate the physical implications of the "semiclassical" model described above as far as the dynamics of localized structures (solitary waves) is concerned, and to provide a critical comparison with the by now widely explored classical model. We adopt a Sagdeev ${ }^{22}$ pseudopotential approach, which has the advantage of allowing potentially larger amplitudes, with clear physical limitations on these. To the contrary, all nonlinear treatments based on a reductive perturbation method can only deal with weaker amplitudes, a constraint imposed by the use of stretched independent variables and of expansions of the dependent variables. Several authors limit themselves to reductive perturbation methods ${ }^{1,7,9,11,12}$ leading to a Korteweg-de Vries (KdV), a nonlinear Schrödinger (NLS), or a Zakharov-Kuznetsov (ZK) evolution equation, the latter for magnetized plasmas.

The semiclassical model discussed here, adopted in a number of works published in the past,,$^{1,4,7,9,11-13}$ has won its place in the current literature mostly thanks to its analytical tractability and despite, admittedly, a certain lack of realism. It might be argued that its simple analytical structure fails to capture the rich dynamics of ultrarelativistic plasmas in the electrostatic approximation, where a more elaborate description may be required. Indeed, more sophisticated models have been proposed in the past (see, e.g., the discussion in Ref. 10 and elsewhere ${ }^{15-18}$ ), in account of localized forms, e.g., potential pulses and bipolar electric fields, such as the ones presented in our study. Our aim here is to discuss the properties and implication of the widely used semiclassical model; hence, discussing more complex (and arguably more realistic) models ${ }^{10}$ lies beyond our scope.

The paper is structured as follows. After this introduction, Sec. II describes the ultrarelativistic e-p-i plasma model, in which the pressure-density relations for the electrons and positrons have exponents $\gamma_{e}=\gamma_{p}=4 / 3,{ }^{1,4,7-9,11,12}$ and recalls the essentials of a Sagdeev pseudopotential analysis for larger-amplitude waves and solitons. Section III is devoted to a numerical evaluation of possible existence ranges in a parametric assessment of where solitons might be encountered, and includes examples of suitable Sagdeev pseudopotentials, soliton profiles, and the associated electric fields. The latter are often more amenable to observations in heliospheric surroundings than the underlying electrostatic soliton potentials on which the theoretical treatment is based. The contrasting classical nonrelativistic formalism with Boltzmann electron and positron distributions $\left(\gamma_{e}=\gamma_{p}=1\right)$ is addressed in Sec. IV, leading to rather unexpected conclusions. For the sake of completeness, the weak-amplitude expansion of the Sagdeev pseudopotentials is given in Sec. V to indicate how results would compare had we started from a reductive perturbation approach. Section VI then summarizes our findings.

\section{ULTRARELATIVISTIC PLASMA MODEL AND SAGDEEV PSEUDOPOTENTIAL METHOD}

The plasma model considered here is composed of warm positive ions in the presence of ultrarelativistic electrons and positrons. ${ }^{1,4,7-9,11,12}$

The properties of the different species' densities are recalled briefly. The warm positive ions are described by the customary fluid continuity and momentum equations, written in a reference frame moving with the solitary structure, so that the time derivatives are transformed away. This choice presupposes that such structures can be found and the available literature on solitary waves abundantly shows that this is a realistic assumption. However, the soliton speeds are as yet unknown and have to be determined at a later stage.

In normalized variables, this gives

$$
\begin{gathered}
\frac{d}{d x}\left(n_{i} u_{i}\right)=0, \\
u_{i} \frac{d u_{i}}{d x}+\beta n_{i} \frac{d n_{i}}{d x}+\frac{d \varphi}{d x}=0 .
\end{gathered}
$$

Densities $n_{i}, n_{e}$, and $n_{p}$, where the labels $i, e$, and $p$ refer to the (positive singly charged) ions, electrons, and positrons, have been normalized to their original (physical) equilibrium densities $n_{i 0}, n_{e 0}$, and $n_{p 0}$, respectively. For the ions, the usual adiabatic pressure-density relations $P_{i} \propto n_{i}^{3}$ have been adopted. Fluid velocities have been normalized with respect to a characteristic ion-acoustic speed $c_{i a}=\sqrt{T_{e} / m_{i}}$, where $T_{e}$ is the kinetic electron temperature (incorporating Boltzmann's constant $k_{B}$ ) and $m_{i}$ the ion mass. Note that $Z_{i}=1$ was assumed for the ion charge state (in fact, a different choice of value, here, could easily be absorbed in the scaling). Furthermore, $\beta=T_{i} / T_{e}$, the electrostatic potential $\varphi$ has been normalized by $T_{e} / e$, and $T_{i}$ includes the factor 3 characterizing adiabatic pressures. Finally, the space coordinate $x$ has been normalized by the electron Debye length $\lambda_{D e}=\left(\varepsilon_{0} T_{e} / n_{e 0} e^{2}\right)^{1 / 2}$.

Integrating (1) with zero boundary conditions far away from the nonlinear structure gives the standard conservation of mass as

$$
n_{i} u_{i}=M,
$$

where $M=V / c_{i a}$ is a measure for the Mach number and $V$ is the velocity the solitary structure would have in an inertial frame.

At this stage, it is worth emphasizing that the choice of zero integration constants far from the nonlinear wave is typical for solitary structures and will also be used for subsequent integrations, for internal consistency. Alas, that automatically precludes any discussion of periodic structures, as can sometimes be encountered in the literature, where after obtaining the Sagdeev pseudopotential a nonzero constant is added, contradicting steps in the derivation.

Using (3) in (2) to eliminate $u_{i}$ yields after integration a biquadratic equation in $n_{i}$, which can be solved for $n_{i}$ in terms of $\varphi$ as ${ }^{23}$

$$
n_{i}=\frac{1}{2 \sqrt{\beta}}\left[\sqrt{(M+\sqrt{\beta})^{2}-2 \varphi}-\sqrt{(M-\sqrt{\beta})^{2}-2 \varphi}\right] .
$$

Because the ions are the inertial species, their thermal velocities have to be smaller than the solitary wave speed, which translates as $\beta<M^{2}$; in other words, the ions are supersonic in the fluid-dynamical parlance. ${ }^{24,25}$ As $M+\sqrt{\beta}>M-\sqrt{\beta}>0$, the ion density ceases to be real if $(M-\sqrt{\beta})^{2}<2 \varphi$. For given parameters $M$ and $\beta$, there is a positive upper limit on the electrostatic potential 


$$
\varphi_{\ell i}=\frac{1}{2}(M-\sqrt{\beta})^{2} .
$$

For ultrarelativistic degenerate electrons and positrons, the equations of state are given by $P_{e} \propto n_{e}^{4 / 3}$ and $P_{p} \propto n_{p}^{4 / 3}$, with the same mass $m_{e}=m_{p}$. In principle, the polytropic pressure-density relations for the hot inertialess species do not really define their temperatures, so that the relations $P_{e}=n_{e} T_{e}$ and $P_{p}=n_{p} T_{p}$ are used, which correspond to the formal definition of the thermal velocities in ordinary fluids as $\sqrt{T_{e} / m_{e}}$ and $\sqrt{T_{p} / m_{p}}$. Consequently, $T_{e} \propto n_{e 0}^{1 / 3}$ and $T_{p} \propto n_{p 0}^{1 / 3}$. Assuming the same Fermi temperature $T_{F e}$ hidden in the proportionality factor then yields

$$
\frac{T_{p}}{T_{e}}=\left(\frac{n_{p 0}}{n_{e 0}}\right)^{1 / 3}=f^{1 / 3},
$$

where $f=n_{p 0} / n_{e 0}$ is the relative fraction of the positive charge residing on the positrons. The electron and positron densities thus become

$$
\begin{gathered}
n_{e}=\left(1+\frac{\varphi}{3}\right)^{3}, \\
n_{p}=\left(1-\frac{\varphi}{3 f^{1 / 3}}\right)^{3},
\end{gathered}
$$

normalized to their own equilibrium densities. This yields additional constraints on $\varphi$, on the positive side due to the positrons, and on the negative side due to the electrons

$$
\begin{gathered}
\varphi_{\ell p}=3 f^{1 / 3}, \\
\varphi_{\ell e}=-3 .
\end{gathered}
$$

It will later be seen that in the ranges of interest, the electron limit plays no role as the model only supports positive solitons. For those, there is a tiny range up to $f=0.081$ where the positron limit intervenes before the ion limit pitches in. Hence, for most of the $f$ ranges of interest, it is the ion limit which ends the available soliton amplitude ranges.

The densities are coupled in the Poisson's equation, written in a normalized form as

$$
\begin{aligned}
& \frac{d^{2} \varphi}{d x^{2}}+\frac{1-f}{2 \sqrt{\beta}}\left[\sqrt{(M+\sqrt{\beta})^{2}-2 \varphi}-\sqrt{(M-\sqrt{\beta})^{2}-2 \varphi}\right] \\
& +f\left(1-\frac{\varphi}{3 f^{1 / 3}}\right)^{3}-\left(1+\frac{\varphi}{3}\right)^{3}=0
\end{aligned}
$$

Integration of (11) gives an energy relation

$$
\frac{1}{2}\left(\frac{d \varphi}{d x}\right)^{2}+S(\varphi, M)=0,
$$

where $S(\varphi, M)$ is the Sagdeev pseudopotential ${ }^{22}$

$$
\begin{aligned}
S(\varphi, M)= & \frac{1-f}{6 \sqrt{\beta}}\left\{\left(6 M^{2}+2 \beta\right) \sqrt{\beta}-\left[(M+\sqrt{\beta})^{2}-2 \varphi\right]^{3 / 2}\right. \\
& \left.+\left[(M-\sqrt{\beta})^{2}-2 \varphi\right]^{3 / 2}\right\}+\frac{3 f^{4 / 3}}{4}\left[1-\left(1-\frac{\varphi}{3 f^{1 / 3}}\right)^{4}\right] \\
& +\frac{3}{4}\left[1-\left(1+\frac{\varphi}{3}\right)^{4}\right] .
\end{aligned}
$$

The Sagdeev pseudopotential for this problem contains two compositional parameters, the ion temperature ratio $\beta$, and the fractional positive density $f$ of the positrons. For simplicity of notation, these parameters are not written explicitly as arguments of $S$, in contrast to $\varphi$ and $M$, which are variables to be determined in order to obtain particular types of solutions of (12), as will be seen below.

Before that, however, we point out that the limit $f=1$ is not allowed from a methodological point of view because in the absence of the ions, there is no inertial species to sustain the waves and solitons. The electrons and positrons are inertialess and can only contribute to the pressure.

Because of the chosen normalization, $f$ plays a dual role, in that it is not only the density ratio of the positrons vs the electrons, but it also determines the positron/electron temperature ratio; cf. (6). The limit $f=0$ is legitimate in terms of the relative densities but perhaps not relevant in the ultrarelativistic e-p-i plasma model as it reduces the model to the special case of ions and electrons only. However, we need in (11) to first take out the positron contribution, and only then let $f \rightarrow 0$. The reason relates to the dual interpretation of $f$, which causes $\lim _{f \rightarrow 0} f n_{p} \neq 0$ and might give spurious terms in (13) when blindly taking the limit $f=0$. Thus, the range for very small but nonzero $f$ needs subtle care and is best avoided.

In order to find (nonperiodic) solitary structures, the origin $\varphi=0$ needs to correspond to an unstable equilibrium; hence, $S(0, M)=S^{\prime}(0, M)=0$ and $S^{\prime \prime}(0, M)<0$, where the primes denote derivatives of $S(\varphi, M)$ with respect to $\varphi$. The integration from (11) to (12) and (13) has also been performed with zero boundary conditions far away from the nonlinear structures.

Defining the acoustic Mach number $M_{s}$ as a solution $M$ of $S^{\prime \prime}(0, M)=0$, it is here given as

$$
M_{s}=\sqrt{\beta+\frac{1-f}{1+f^{2 / 3}}} .
$$

The requirement for solitary waves, that $S^{\prime \prime}(0, M)<0$, translates into $M>M_{s}$. In cases where one might obtain waves at the acoustic speed, these inequalities become $S^{\prime \prime}(0, M) \leqslant 0$ and $M \geqslant M_{s}$, but acoustic speed solitons are not possible in the present model, as seen below. At given $f, M_{s}$ increases as $\beta$ increases. On the other hand, since $1-f \leqslant 1+f^{2 / 3}$, the fraction $(1-f) /\left(1+f^{2 / 3}\right)$ is 1 at $f=0$ and 0 at $f=1$, and thus decreases monotonically at increasing $f$, as can easily be checked. At given $\beta, M_{s}$ decreases as $f$ increases.

It is interesting to point out that expression (14) physically represents the true sound speed in the plasma dynamical system under consideration, i.e., it gives the phase speed of ion-acoustic waves in the long wavelength limit. Indeed, had we considered the time dependent fluid model in the reference frame (anticipating small amplitude harmonic solutions), we would have derived a linear dispersion relation in the form

$$
\omega^{2}=\beta k^{2}+\frac{(1-f) k^{2}}{k^{2}+1+f^{2 / 3}} .
$$

As one may verify after a straightforward calculation, upon evaluating the limit $\lim _{k \rightarrow 0}(\omega / k)$, one finds precisely the right-hand side of (14), thus corroborating our result for the acoustic Mach number threshold (lower bound). of 


$$
\begin{aligned}
S^{\prime \prime \prime}\left(0, M_{s}\right)= & \frac{7\left(1+f^{2 / 3}\right)^{2}+2 f^{1 / 3}\left(1+f^{1 / 3}\right)^{2}}{3(1-f)} \\
& +4 \beta \frac{\left(1+f^{2 / 3}\right)^{3}}{(1-f)^{2}}>0 .
\end{aligned}
$$

The sign of $S^{\prime \prime \prime}\left(0, M_{s}\right)$ gives the polarity (sign of $\left.\varphi\right)$ of the KdV-like solitons, here, positive. KdV-like solitons mean that their amplitudes shrink to zero as $M \rightarrow M_{s}$, ${ }^{27}$ as structures obtained though reductive perturbation analysis do, but with amplitudes that can grow larger than admissible under an iterative expansion scheme. If, in addition, solitons of the opposite polarity would also occur, for specific parameter regimes, they must be nonKdV-like, i.e., their amplitudes would have to be finite. ${ }^{27}$ Here, however, nonKdV-like (negative) solitons were not found in our numerical evaluations.

Further remarks are that the mathematics of the model described here can easily be adapted to negative instead of positive ions, by simply changing the sign of $\varphi$, interchanging the roles of the electrons and positrons, and adapting the normalization and interpretation of the parameters accordingly. This is chiefly due to the equal masses and opposite charges of electrons and positrons, and the assumption of their having equal Fermi temperatures.

\section{EXISTENCE DOMAINS, SOLITON, AND ELECTRIC FIELD PROFILES}

After having discussed and finished the analytic description of the model, we come to the numerical evaluation of the Sagdeev pseudopotential and its solutions. The procedure is as follows. The plasma model is (numerically) defined by specifying the compositional parameters $f$ and $\beta$. Within this setting, the problem is to find possible $M$ such that solitons can be generated and then determine their amplitudes and polarities. This leads us in a logical way to existence diagrams for $M$ as functions of $f$ and $\beta$.

It is clear that $M_{s}$ gives the minimum values of $M$. Maximum values $M_{\text {max }}$ can be obtained by imposing the reality conditions on the species' densities. It will turn out that the solitons have positive polarities and that for $f>0.081$ and $\beta=0$ the condition $\varphi \leqslant \varphi_{\ell i}$ is reached before $\varphi_{\ell p}$ pitches in. For $f$ strictly zero, $\varphi_{\ell p}$ plays no role, and for $0<f<0.081$ the amplitudes are limited by $\varphi_{\ell p}$. Putting the maximum roots at $\varphi_{\ell p}$ or $\varphi_{\ell i}$ means that $M=M_{\text {max }}$ is a solution of $S\left(\varphi_{\ell p}, M_{\max }\right)$ $=0$ or $S\left(\varphi_{\ell i}, M_{\max }\right)=0$, depending on the $f$ range considered. Unfortunately, whereas we have an analytical expression for $M_{s}$, the corresponding values for $M_{\max }$ have to be obtained numerically.

Existence domains can be visualized in various ways. The most obvious way is by first plotting $M_{s}$ and $M_{\max }$ as functions of $f$ for given values of $\beta$, as shown in Fig. 1. Although we have for future reference included also curves relating to the Boltzmann description of the hot species, these will be discussed in Sec. IV. In the remainder of this section, we evaluate the properties for ultrarelativistic electrons and positrons.

Thus, the maximum values $M_{\max }$ are obtained by evaluating (13) for $\varphi=\varphi_{\ell p}$ or $\varphi=\varphi_{\ell i}$, fixing $\beta$, and solving $S\left(\varphi_{\ell p}, M\right)=0$ or $S\left(\varphi_{\ell \text { i }}\right.$, $M)=0$ for $M$ as function of $f$. We have considered only two relevant values for $\beta$ to distinguish between warm $(\beta=0.1)$ and strictly cold $(\beta$ $=0$ ) ions. Wanting to maintain a clear separation between the ion and the hot species temperatures, we have not considered higher values of $\beta$, but these might easily be included, working along similar lines.

The range in $M$ between minimum and maximum decreases as $f$ increases. For cold ions, with $\beta=0$, the existence curves start at lower

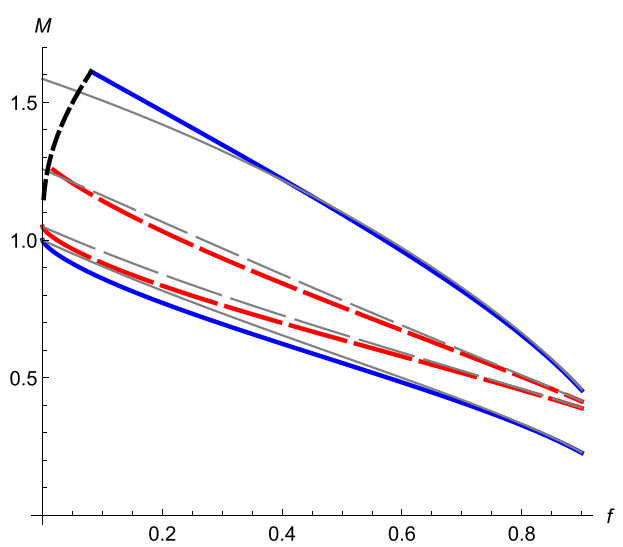

FIG. 1. Existence domains for minimum and maximum $M$ as a function of $f$, for values of $\beta=0$ (blue or dotted black) and 0.1 (red dashed or dotted black). In each pair of curves, lower curves are for $M_{s}$ and upper curves for $M_{\max }$. The black curve is for $\varphi_{\ell p}$ as a limiting factor, at small nonzero $f$, the blue or red dashed upper curves when $\varphi_{\ell i}$ is reached before $\varphi_{\ell p}$. For future comparison, the gray curves refer to Boltzmann descriptions and will be discussed in Sec. IV. Note that the expected lower (acoustic) and upper (infinite compression) limit values (namely, $M_{\min }=1$ and $M_{\max } \simeq 1.58$ ) are recovered for $f=\beta=0$ in the classical Boltzmann case (continuous gray curves), as anticipated for electron-(cold-)ion plasmas. ${ }^{22}$

$M_{s}$ than for $\beta=0.1$. The converse happens for $M_{\max }$ because the upper limit $\varphi_{\ell i}$ decreases with increasing $\beta$, besides the influence of $f$. As is well known for Sagdeev pseudopotentials, at given compositional parameters the curves for varying $M$ cannot cross, so that increasing $M$ implies increasing amplitudes $\varphi .^{26}$ The deeper bulge in the Sagdeev pseudopotential then causes the larger solitons to be narrower.

Another way of illustrating the existence domains for potential solitons is shown in Fig. 2 to see how the amplitudes of the solutions vary with $M$, for given values of $\beta$ and $f$. This has been done for $\beta=0$ (higher curves) and $\beta=0.1$ (lower curves), for different values of $f=0$ (yellow long-dashed), 0.3 (green dotted-dashed), 0.6 (red dashed), and 0.9 (blue). The curves start for $\varphi=0$ at the respective $M_{s}$, and all end at the corresponding $\varphi_{\ell i}$.

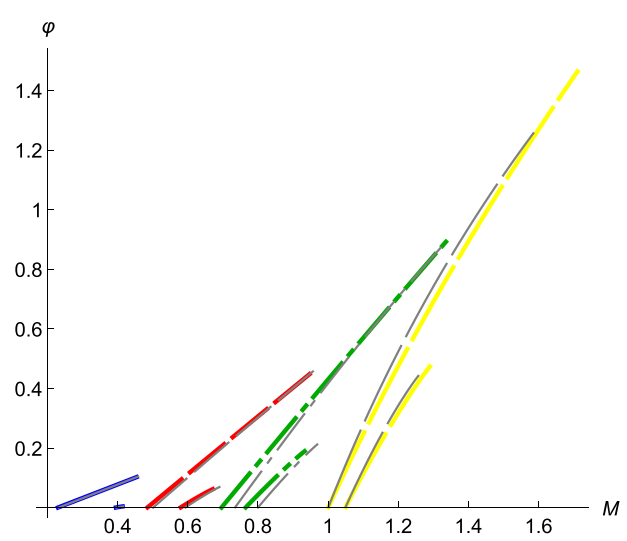

FIG. 2. Existence domain for $\varphi$ as a function of $M$, for different values of $f=0$ (yellow long-dashed), 0.3 (green dotted-dashed), 0.6 (red dashed), and 0.9 (blue). In each pair of curves, higher curves are for $\beta=0$, lower curves for $\beta=0.1$. The curves start for $\varphi=0$ from the respective $M_{s}$ and end at the corresponding $\varphi_{\ell i}$. Gray curves refer to the Boltzmann model to be discussed in Sec. IV. 
The fact that all the curves in Fig. 2 start for $M_{s}$ at $\varphi=0$ is an indication that there are no solitons at the acoustic speed, for which a finite minimal amplitude would be required. Another remark is that the existence range for $f=0.9$ and $\beta=0.1$ is tiny, at the limit of numerical accuracy, so that it will not be addressed further. This reminds us again that the limit $f \rightarrow 1$ eliminates the (adiabatic or cold) ions, so that there can no longer be acoustic-type modes, as the remaining species do not carry inertia.

Taking these indications together, it is seen that there is quite a range in parameter space for which solitons can be obtained. Moreover, the highest amplitudes and Mach numbers occur in the absence of positrons and for cold ions. We repeat again that we will first choose values for $f$ and $\beta$, and for those we determine the appropriate $M$ and $\varphi$. However, in several papers in the literature, one picks one or more values for $M$ and then looks for suitable compositional parameters. This looks like putting the cart before the horses, as we know from experience that for a chosen set of compositional parameters like $f$ and $\beta$, there is a family of Sagdeev pseudopotentials varying with $M$, which leads to a range of soliton amplitudes, intimately correlated with a range of Mach numbers.

We give some examples of Sagdeev pseudopotentials in Fig. 3, for sets of curves: blue dashed $(f=0.2, M=1)$, green long-dashed $(f=0.5$, $M=0.75)$, and red solid $(f=0.8, M=0.5)$. In each pair of curves with the same coding, the thin curves are for $\beta=0$ and the thick ones for $\beta$ $=0.1$. The Mach numbers have been adjusted so that for each pair the same $M$ can be used, with the help of Fig. 1.

As long as the boundaries of the existence diagrams in Fig. 1 are heeded, one can pick parameters and plot the relevant Sagdeev pseudopotentials, as many as is needed, suggested, wanted. This, however, will always lead to similar plots, which are not included here, to avoid repetitious diagrams.

Once the Sagdeev potentials are found, one can plot the soliton and electric profiles associated with their nonzero root. In Figs. 4 and 5, we show how such profiles look. The same color coding is used as in Fig. 3, but for graphical clarity, the lines have been drawn at equal thickness, and the larger amplitudes in each pair correspond to $\beta=0$, the smaller to $\beta=0.1$.

After the soliton profiles are given in Fig. 4, one can determine their associated electric field profiles in Fig. 5, based on $E=-d \varphi / d x$.

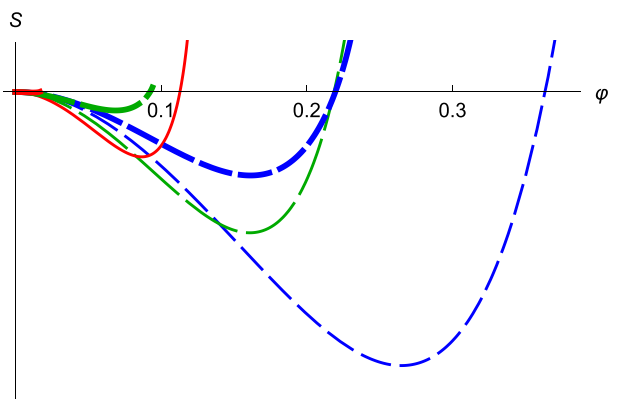

FIG. 3. Sagdeev pseudopotentials: Blue dashed curves for $f=0.2$ and $M=1$, green long-dashed curves for $f=0.5$ and $M=0.75$, and red solid curves for $f=0.8$ and $M=0.5$. In each pair of curves with the same coding, the thin curves are for $\beta=0$ and the thick ones for $\beta=0.1$.

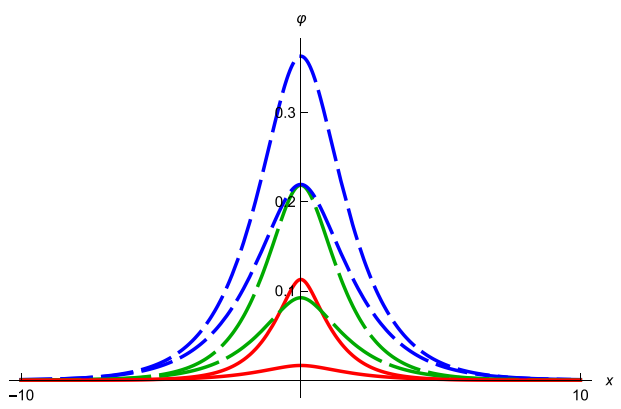

FIG. 4. Soliton profiles for the solutions of the Sagdeev pseudopotentials shown in Fig. 3, with the same color coding. The larger amplitudes in each pair correspond to $\beta=0$, the smaller to $\beta=0.1$.

\section{BOLTZMANN ELECTRONS AND POSITRONS}

At the opposite end from ultrarelativistic electrons and positrons, we find in the classical nonrelativistic case Boltzmann distributions, rather than the adiabatic pressure-density relations used or discussed in many papers in the literature. ${ }^{1,2,4-6,9,12}$ The correctness of Boltzmann distributions for the hot species has been argued recently ${ }^{20}$ because their thermal speed is very much greater than the wave speed (the ratio approaching infinity). As a result, during the wave motion, heat flow between the particles locally involved in the wave and those that are a long way away, in the undisturbed region of the plasma, can take place "instantaneously." Precisely that requirement is reflected in the basic isothermal assumption underpinning Boltzmann distributions.

Within this model, the ion characteristics and densities are not affected, but the electron and positron densities become

$$
\begin{gathered}
n_{e}=\exp (\varphi), \\
n_{p}=\exp (-\varphi),
\end{gathered}
$$

instead of (7) and (8). These are now well-defined for all $\varphi$, so that there are no density constraints on $\varphi$, other than $\varphi_{\ell i}$. Because in the ultrarelativistic description, the electrons and positrons were assumed to have equal Fermi temperatures, we have in (17) and (18) also chosen equal (classical) temperatures.

The densities are coupled in the Poisson's equation, written in a normalized form as

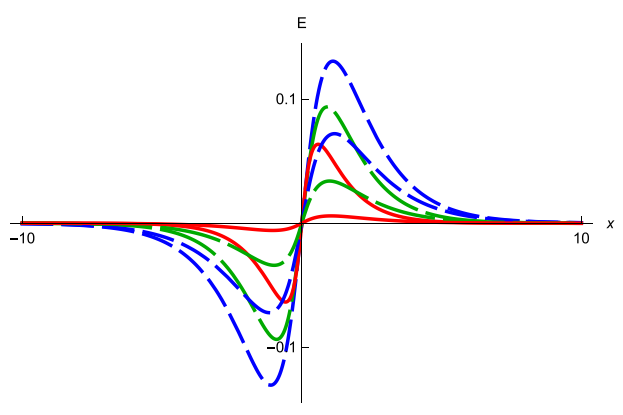

FIG. 5. Electric field profiles of the solitons shown in Fig. 4, with the same conventions. 


$$
\begin{aligned}
& \frac{d^{2} \varphi}{d x^{2}}+\frac{1-f}{2 \sqrt{\beta}}\left[\sqrt{(M+\sqrt{\beta})^{2}-2 \varphi}-\sqrt{(M-\sqrt{\beta})^{2}-2 \varphi}\right] \\
& \quad+f \exp (-\varphi)-\exp (\varphi)=0 .
\end{aligned}
$$

Here, $f=n_{p 0} / n_{e 0}$ and does not refer to the (equal) temperatures. Integration of (19) involves a new Sagdeev pseudopotential

$$
\begin{aligned}
S(\varphi, M)= & \frac{1-f}{6 \sqrt{\beta}}\left\{\left(6 M^{2}+2 \beta\right) \sqrt{\beta}-\left[(M+\sqrt{\beta})^{2}-2 \varphi\right]^{3 / 2}\right. \\
& \left.+\left[(M-\sqrt{\beta})^{2}-2 \varphi\right]^{3 / 2}\right\} \\
& +f[1-\exp (-\varphi)]+[1-\exp (\varphi)] .
\end{aligned}
$$

Performing the same analysis as in Sec. II leads first to

$$
M_{s}=\sqrt{\beta+\frac{1-f}{1+f}},
$$

and

$$
S^{\prime \prime \prime}\left(0, M_{s}\right)=\frac{2\left(1+4 f+f^{2}\right)}{1-f}+4 \beta \frac{(1+f)^{3}}{(1-f)^{2}} .
$$

Expression (22) is positive at all admissible $f$ and $\beta$ also in the Boltzmann case, and these solitons will have positive polarity.

All this leads to the gray curves in Figs. 1 and 2, which are very similar and close to the curves obtained in the ultrarelativistic case. This is all the more remarkable, given that the physics of the hot species is completely different, at the opposite end of the range.

We now turn to examples of Sagdeev pseudopotentials with Boltzmann electrons and positrons in Fig. 6. In each pair of curves with the same coding, the thin curves are for $\beta=0$ and the thick ones for $\beta=0.1$. The Mach numbers have been adjusted so that for each pair the same $M$ is used, with the help of Fig. 1.

As long as the boundaries of the existence diagrams in Fig. 1 are obeyed, one can again pick parameters and plot the relevant Sagdeev pseudopotentials.

Once the Sagdeev potentials are found, one can plot the soliton and electric profiles, all running parallel to what happened in Sec. III. In Figs. 7 and 8, we show how their profiles look. The same color

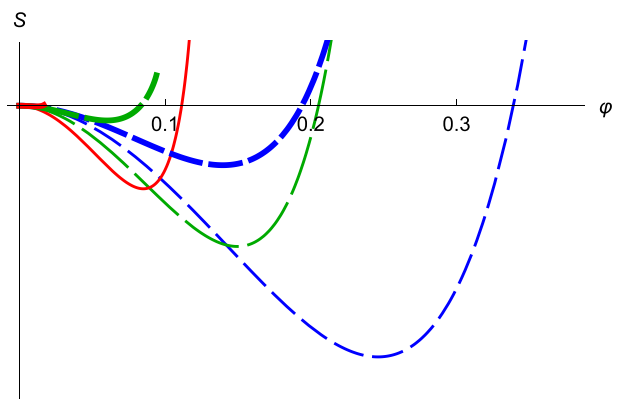

FIG. 6. Sagdeev pseudopotentials for Boltzmann electrons and positrons: Blue dashed curves for $f=0.2$ and $M=1$, green long-dashed curves for $f=0.5$ and $M=0.75$, and red solid curves for $f=0.8$ and $M=0.5$. In each pair of curves with the same coding, the thin curves are for $\beta=0$, and the thick ones for $\beta=0.1$.

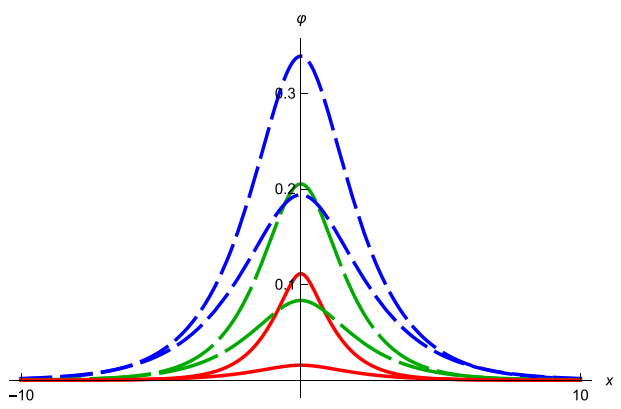

FIG. 7. Soliton profiles for the solutions of the Sagdeev pseudopotentials shown in Fig. 6, with the same color and line coding. The larger amplitudes in each pair correspond to $\beta=0$, the smaller to $\beta=0.1$.

coding is used as in Fig. 6, but for graphical clarity the lines have been drawn at equal thickness, and the larger amplitudes in each pair correspond to $\beta=0$, the smaller to $\beta=0.1$.

From the soliton profiles given in Fig. 7, one determines their associated electric field profiles shown in Fig. 8, through $E=-d \varphi / d x$.

A comparison between the ultrarelativistic and Boltzmann descriptions for the electrons and positrons is shown in Figs. 9 and 10, for Sagdeev pseudopotentials and their soliton profiles. This is illustrated for the same parameters $f=0.2, \beta=0$, and $M=1$ because the existence diagrams clearly indicate that for small $f$ and $\beta=0$ the strongest solitons are obtained, at given $M$. Other parameters being equal, ultrarelativistic electrons and positrons produce slightly larger and slightly narrower solitons than Boltzmann electrons and positrons, but the differences are of minor importance, only quantitative.

This remains a surprising result, given the vastly different environment and thermodynamic description.

\section{KORTEWEG-DE VRIES SOLITONS}

For the sake of comparison, we briefly look at weakly nonlinear solitons, which can be obtained in two different ways. One is to do the complete reductive perturbation analysis, by stretching in the usual way the independent variables, here space and time, and expanding the dependent variables to the lowest relevant nonlinear order. This procedure yields in the generic case the well-known Korteweg-de Vries (KdV) equation, which has become quite popular and arguably one of the most studied nonlinear evolution equations. The reason is that the procedure is algorithmically straightforward and applies to a

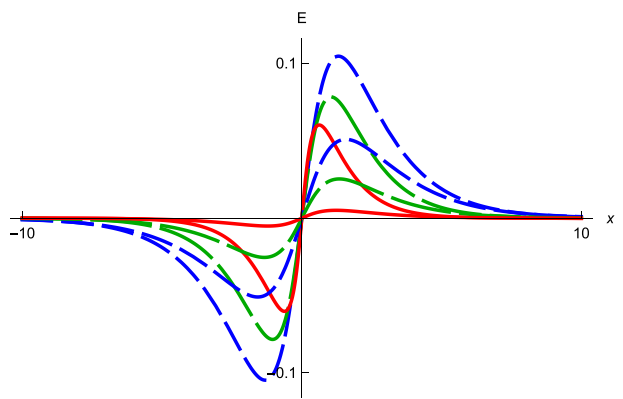

FIG. 8. Electric field profiles of the solitons shown in Fig. 7, with the same conventions. 


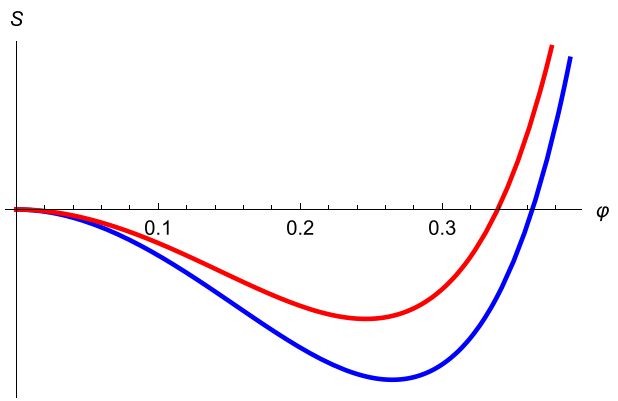

FIG. 9. Example of Sagdeev pseudopotentials for $f=0.2, \beta=0$, and $M=1$, in the ultrarelativistic (blue) and Boltzmann (red) descriptions for the hot species.

great many physical situations. Its drawback is that the truncation of the nonlinearities, in principle to second order, limits the applicability to weakly nonlinear waves and solitons although there is no real quantitative criterion to determine how small the weakly nonlinear waves have to be for the KdV equation to be a valid description.

In investigations where one has already obtained the Sagdeev pseudopotential, as in this paper, and if the aim is the obtention of a traveling wave solution, it is easier to expand the Sagdeev pseudopotential. This leads to

$$
S(\varphi, M)=\frac{1}{2} S^{\prime \prime}(0, M) \varphi^{2}+\frac{1}{6} S^{\prime \prime \prime}(0, M) \varphi^{3},
$$

since the first two terms in the expansion vanish at all $M$ by choice of integration constants and charge neutrality at equilibrium. Furthermore, we know that the solitons are superacoustic; hence, we will expand $S^{\prime \prime}(0, M)$ for $M=M_{s}+\mu$, up to first order in $\mu$, to get for the ultrarelativistic plasma model that

$$
A=S^{\prime \prime}\left(0, M_{s}+\mu\right) \simeq-\frac{\left(1+f^{2 / 3}\right)^{3 / 2} \sqrt{1-f+\beta\left(1+f^{2 / 3}\right)}}{1-f} \mu,
$$

and in the case of Boltzmann hot species that

$$
A=S^{\prime \prime}\left(0, M_{s}+\mu\right) \simeq-\frac{(1+f)^{3 / 2} \sqrt{1-f+\beta(1+f)}}{1-f} \mu .
$$

In $S^{\prime \prime \prime}(0, M)$, we replace $M$ by $M_{s}$ to balance both terms on the right hand side of $(23)$ in the sense that $\mu \varphi^{2} \sim \varphi^{3}$ are on the same

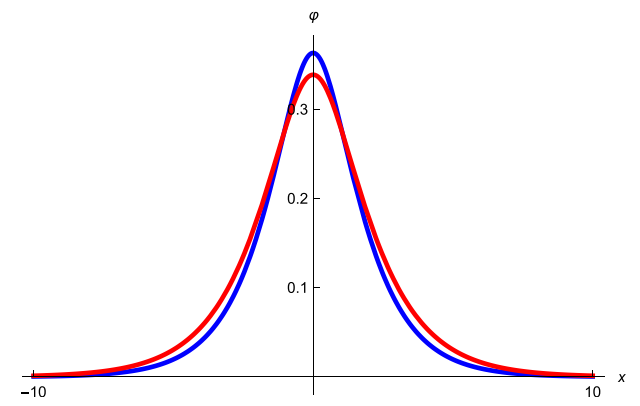

FIG. 10. Soliton profiles for the Sagdeev pseudopotentials shown in Fig. 9, with the same parameters and curve coding. order of smallness. Moreover, the expressions for $S^{\prime \prime \prime}\left(0, M_{s}\right)$ have already been obtained before, in (16) and (22), in the context of the determination of the soliton polarities. Calling now $B=S^{\prime \prime \prime}\left(0, M_{s}\right)$ allows us to write the typical sech squared $\mathrm{KdV}$-soliton solution as

$$
\varphi=\frac{3|A|}{B} \operatorname{sech}^{2}\left(\frac{1}{2} \sqrt{|A|} x\right) .
$$

In our description, $x$ is already a comoving coordinate. Since $A$ is linear in $\mu$, the degree of superacousticity determines not only the amplitude but also the inverse width.

Not wanting to dwell too long on the KdV solutions to the problem investigated in this paper, we will only show in Fig. 11 specific examples of (23), where the amplitudes have been adjusted to correspond to the roots of the Sagdeev pseudopotentials illustrated in Fig. 9. This is probably stretching the applicability of the KdV description beyond its small-amplitude assumptions, but still generates pseudopotentials and soliton profiles which are very similar to what was obtained before. Due to the weaker nonlinearities in the KdV description, the bulges in the Sagdeev pseudopotentials are not as deep as in the fully nonlinear ultrarelativistic or Boltzmann treatments, for the same soliton amplitudes. The solutions given by (26) are illustrated in Fig. 12, in comparison to earlier ultrarelativistic and Boltzmann profiles, so they show the uncanny similarity.

\section{CONCLUSIONS}

In this paper, we have first looked at ion-acoustic solitons in a specific semiclassical plasma model with ultrarelativistic electrons and positrons in the presence of fluid adiabatic (classical) ions. After having derived the theoretical Sagdeev pseudopotential framework for the description of large-amplitude nonlinear solutions, we have done a parametric investigation to establish the existence regions in terms of fractional densities, temperature ratios, and soliton speeds. Once these are known, many examples of pseudopotentials, soliton, and electric field profiles can be generated numerically from the analytical results. Of those, only some specific illustrations have been included.

For the sake of comparison, we have also investigated the classical nonrelativistic counterpart. While this has in the literature been through adiabatic pressure-density relations for the hot species, recent work has established that in this case the hot species need to be described by Boltzmann distributions. Thus, our classical results differ

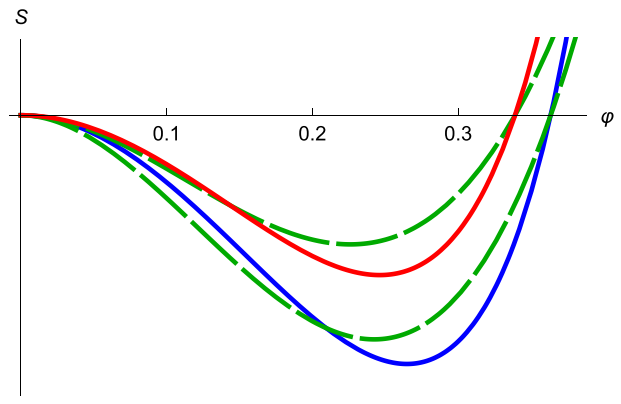

FIG. 11. Example of KdV Sagdeev pseudopotentials for $f=0.2$ and $\beta=0$ (green dashed curves), superimposed on ultrarelativistic (blue) and Boltzmann (red) results shown in Fig. 9. Here, the superacoustic velocity increment $\mu$ has been adjusted to give precisely the same amplitudes. 


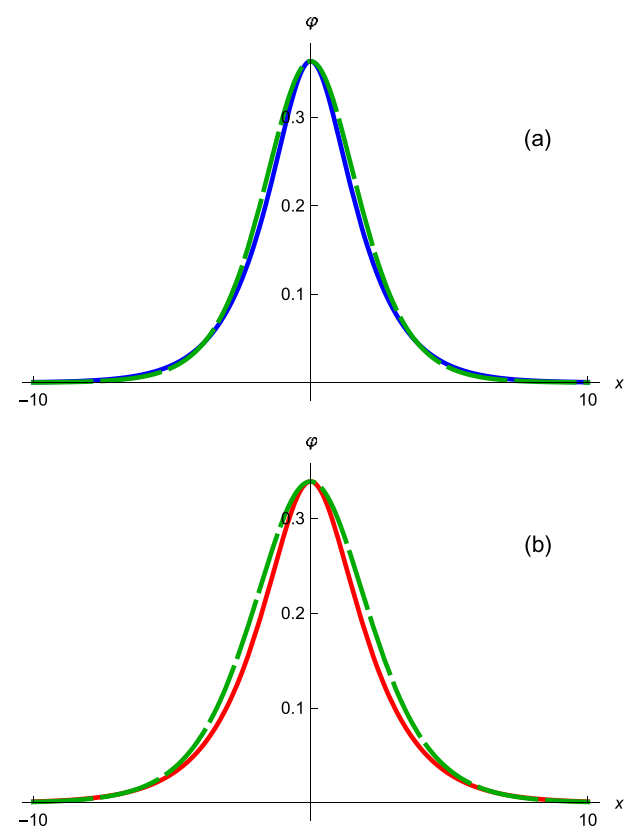

FIG. 12. Soliton profiles for the Sagdeev pseudopotentials shown in Fig. 11, with the same parameters and curve coding: (a) blue for ultrarelativistic and (b) red for Boltzmann, and dashed green for corresponding $\mathrm{KdV}$ at same amplitudes.

qualitatively from what is available in the literature. To our great surprise, for the same parameter choices, the soliton and electric field profiles show scant differences between the ultrarelativistic and the classical nonrelativistic Boltzmann modeling even though the physical description of the hot species is radically different, at the opposite ends of the possible behavior.

We have also included a short comparison between the fully nonlinear Sagdeev pseudopotential description and the weaker-amplitude reductive perturbation approach, through the standard sech squared $\mathrm{KdV}$ solitons, where the nonlinearities have been truncated to low powers of the electrostatic potential. Even so, the soliton profiles are not radically different although it could be argued that we have adjusted the excess-over-acoustic velocity to arrange for solitons of equal amplitudes in each comparison, and doing so might have stretched the applicability of the KdV results beyond what is theoretically admissible.
The conclusion that very similar "quantitative" results have been obtained for rather "qualitatively" different physical descriptions of the hot pair species and of the nonlinearities is quite unexpected and leaves an open question, to which no immediate answer seems available.

\section{ACKNOWLEDGMENTS}

I.K. has gratefully acknowledged support from ADEK (Abu Dhabi Department of Education and Knowledge) in the form of an AARE-2018 (ADEK Award for Research Excellence 2018) grant.

\section{REFERENCES}

${ }^{1}$ A. Rasheed, G. Murtaza, and N. L. Tsintsadze, Phys. Rev. E 82, 016403 (2010).

${ }^{2}$ M. Akbari-Moghanjoughi, Phys. Plasmas 18, 012701 (2011).

${ }^{3}$ A. Rasheed, N. L. Tsintsadze, and G. Murtaza, Phys. Plasmas 18, 112701 (2011).

${ }^{4}$ N. Roy, S. Tasnim, and A. A. Mamun, Phys. Plasmas 19, 033705 (2012).

${ }^{5}$ M. Akbari-Moghanjoughi, Phys. Plasmas 19, 064703 (2012).

${ }^{6}$ N. Roy, Phys. Plasmas 19, 064704 (2012).

${ }^{7}$ R. Sabry, W. M. Moslem, and P. K. Shukla, Phys. Plasmas 19, 122903 (2012).

${ }^{8}$ W. M. Moslem, Astrophys. Space Sci. 342, 351 (2012).

${ }^{9}$ S. Sadiq, S. Mahmood, Q. Haque, and M. Z.Ali, Astrophys. J. 793, 27 (2014).

${ }^{10}$ M. McKerr, F. Haas, and I. Kourakis, Phys. Rev. E 90, 033112 (2014).

${ }^{11}$ A.Rahman, M. McKerr, W. F. El-Taibany, I. Kourakis, and A. Qamar, Phys. Plasmas 22, 022305 (2015).

${ }^{12}$ T.-L. Liu, Y.-L. Wang, and Y.-Z. Lu, Chin. Phys. B 24, 025202 (2015).

${ }^{13}$ A.Rahman, I. Kourakis, and A. Qamar, IEEE Trans. Plasma Sci. 43, 974 (2015).

${ }^{14}$ S. Mahmood, S. Sadiq, Q. Haque, and M. Z. Ali, Phys. Plasmas 23, 062308 (2016).

15 I. S. Elkamash, F. Haas, and I. Kourakis, Phys. Plasmas 24, 092119 (2017).

${ }^{16}$ I. Kourakis, M. McKerr, I. S. Elkamash, and F. Haas, Plasma Phys. Controlled Fusion 59, 105013 (2017).

${ }^{17}$ I. S. Elkamash, I. Kourakis, and F. Haas, Phys. Rev. E 96, 043206 (2017).

${ }^{18}$ I. S. Elkamash and I. Kourakis, Phys. Plasmas 25, 052124 (2018).

${ }^{19}$ F. Verheest, Phys. Plasmas 13, 082301 (2006).

${ }^{20}$ M. A. Hellberg, F. Verheest, and R. L. Mace, Phys. Plasmas 25, 032303 (2018).

${ }^{21} \mathrm{G}$. Livadiotis, Astrophys. J. 874, 10 (2019).

${ }^{22}$ R. Z. Sagdeev, in Reviews of Plasma Physics, edited by M. A. Leontovich (Consultants Bureau, New York, 1966), Vol. 4, pp. 23-91.

${ }^{23}$ S. S. Ghosh, K. K. Ghosh, and A. N. Sekar Iyengar, Phys. Plasmas 3, 3939 (1996).

${ }^{24}$ J. F. McKenzie, Phys. Plasmas 9, 800 (2002).

${ }^{25}$ F. Verheest, T. Cattaert, G. S. Lakhina, and S. V. Singh, J. Plasma Phys. 70, 237 (2004).

${ }^{26}$ F. Verheest, Phys. Plasmas 17, 062302 (2010).

${ }^{27}$ F. Verheest, M. A. Hellberg, and T. K. Baluku, Phys. Plasmas 19, 032305 (2012). 